\title{
Insulin resistance with impaired fasting glucose increases the risk of NAFLD
}

\author{
Kristina Önnerhag $^{1 *}$, Peter M. Nilsson ${ }^{2}$, Stefan Lindgren ${ }^{1,2}$ \\ ${ }^{1}$ Department of Gastroenterology and Hepatology, Skåne University Hospital, Malmö, Sweden \\ ${ }^{2}$ Department of Clinical Sciences, Lund University, Skåne University Hospital, Malmö, Sweden \\ Email: ${ }^{*}$ Kristina.Onnerhag@med.lu.se
}

Received 22 May 2013; revised 20 June 2013; accepted 27 June 2013

Copyright (C) 2013 Kristina Önnerhag et al. This is an open access article distributed under the Creative Commons Attribution License, which permits unrestricted use, distribution, and reproduction in any medium, provided the original work is properly cited.

\begin{abstract}
Background: Our aim was to investigate the frequency of elevated liver enzymes and NAFLD in patients with known insulin resistance during 15 years of follow-up. Methods: Subjects with insulin resistance were identified from a population-based prospective cohort study in Sweden, Malmö Diet and Cancer Study, conducted in 1991-1996. In all, 285 non-diabetic subjects with insulin resistance established by HOMA-IR (homeostasis model assessment) were invited to do the liver function testing and if elevated, they should be further assessed by radiological examination of the liver, anthropometric measures and blood testing. Results: 165 subjects (57.9\%) agreed to do the liver function testing. Of these, 25 subjects (15\%) had elevated liver enzymes. After exclusion of other diseases, 5 of the remaining 21 subjects (23.8\%) had radiological signs of steatosis. Liver steatosis significantly correlated with ALT (alanine aminotransferase) $(p=0.04)$, HOMA-IR $(p=0.00)$ and the metabolic syndrome $(p=0.03) .80 \%$ of the subjects with NAFLD had either developed type 2 diabetes mellitus or had impaired fasting glucose and $80 \%$ fulfilled the WHO-criteria for the metabolic syndrome, which were of significant differences to the group without NAFLD. Conclusion: The risk of developing elevated liver enzymes and NAFLD at long-term follow-up in insulin resistant subjects is not insignificant, but mainly associated with the simultaneous development of impaired fasting glucose, established diabetes mellitus and/ or the metabolic syndrome.
\end{abstract}

Keywords: Insulin Resistance; Metabolic Syndrome; NAFLD; Steatosis

"Corresponding author.

\section{INTRODUCTION}

Non-alcoholic fatty liver disease (NAFLD) is one of the leading causes of chronic liver disease in western countries. It is highly prevalent among individuals with obesity, dyslipidemia, diabetes mellitus and insulin resistance, main features of the metabolic syndrome. The prevalence of NAFLD in the general population in Europe varies between $2 \%-46 \%$, and reaches up to $70 \%$ in people with obesity or type 2 diabetes mellitus [1-4]. The prevalence of the metabolic syndrome in adults varies between 5\% - 36\% in Europe depending on the criteria used and is increasing, causing considerable morbidity and mortality. The simultaneous presence of the different features of the metabolic syndrome increases the risk of NAFLD [5-7].

Radiological imaging is often used to diagnose NAFLD. If $\geq 20 \%-30 \%$ of the liver is composed of fat, the sensitivity for detecting liver steatosis is $85 \%$ and specificity 94\% with ultrasound (US) compared to histology, with similar figures for computer tomography (CT) $[8,9]$.

NAFLD is a common cause of asymptomatic elevation of liver enzymes, in the majority of cases alanine aminotransferase (ALT) [1,2], but the entire spectrum of NAFLD can be seen with normal ALT values [10-12].

The histological spectrum of NAFLD ranges from simple steatosis, which seems to have a benign course, to some cases with inflammation (non-alcoholic steatohepatitis, NASH) and cirrhosis. The histological findings are mainly identical to alcoholic liver disease and it is generally agreed that an ethanol intake of 140 grams/week is the maximum allowable level [5,13-17].

Insulin resistance is considered the common pathogenic event linking the metabolic abnormalities with NAFLD, but the causal relation between steatosis and insulin resistance is under debate. Insulin resistance is usually defined as a defect in insulin action resulting in hyperinsulinemia to maintain euglycemia. Visceral adi- 
pose tissue, which is particularly insensitive to insulin, seems to be of major importance, resulting in an increased delivery of free fatty acids (FFA) to the liver [4, 18-21]. The prevalence of insulin resistance in the general population is not entirely known. In one study, 5.1\% of a normal-weight adult population without metabolic disorders were considered insulin resistant, with increasing prevalence if co-existing metabolic disorders [22]. It is well-known that the majority of patients with NAFLD have insulin resistance. However, the risk of an individual with insulin resistance of developing NAFLD is not known.

Our aim was to investigate the frequency of NAFLD in individuals with known insulin resistance and elevated liver enzymes, without overt diabetes mellitus, at longterm follow-up.

\section{PATIENTS AND METHODS}

\subsection{Study Design}

Malmö Diet and Cancer Study (MDC) is a populationbased prospective cohort study which was conducted between 1991 and 1996 in a city in southern Sweden with approximately 240,000 inhabitants at that time. Women born between 1923 and 1950, and men born between 1925 and 1945 were invited to participate. Baseline examination comprised assessment of dietary habits, lifestyle factors, medication and previous and present medical history with a self-reported 141-item questionnaire. Blood samples were drawn and a medical examination conducted. 28,098 participants were examined, 17,035 were women $(60.6 \%$, with a mean age of 57.5 years) and 11,063 were men $(39.4 \%$, with a mean age of 59.3 years).

A subpopulation, a random sample of $50 \%$ of the MDC cohort, consisting of non-diabetic subjects without a history of myocardial infarction, stroke or diabetes mellitus was further examined. Insulin resistance was calculated for each individual as HOMA-IR, the homeostasis model assessment of insulin resistance (fasting insulin $\mathrm{x}$ fasting blood glucose/22.5). Subjects whose values of HOMA-IR exceeded the gender-specific $75^{\text {th }}$ percentile (i.e. 1.80 for women and 2.12 for men) were considered insulin resistant $(n=1189)$. In line with this metabolic disorder insulin resistance was associated with a higher risk of myocardial infarction and death [23].

In a subsequent study, a stratified sampling according to the HOMA-IR levels above was performed. $40 \%$ of the individuals with insulin resistance were invited and re-examined in 1999-2000. Individuals who had developed diabetes mellitus or with missing information were excluded in this study [24].

The insulin resistant subjects without diabetes mellitus who participated in both of the above studies, were iden- tified for our study. After initial exclusion (Figure 1) 285 were invited to blood sampling including liver function tests (LFTs: aspartate aminotransferase (AST), alanine aminotransferase (ALT), gamma-GT, bilirubin, alkaline phosphatase (ALP) and PK-INR). Subjects with elevated LFTs were invited to further assessment including clinical examination, anthropometric measures, blood samples for analysis and radiological examination of the liver (US or CT due to local availability).

To estimate the frequency of diabetes mellitus and NAFLD among all the excluded subjects (Figure 1) we linked their unique personal identification number to the National Board of Health and Welfare's National Patient Register (NPR) for inpatient records of diagnoses, and to the Swedish Cause of Death Register (SCDR) for underlying and multiple causes of death. Diagnoses were coded according to the WHO International Classification of Diseases, ICD-9 (1 January 1977 to 31 December 1993) and ICD-10 (from 1 January 1994).

Finally we retrieved data from the re-evaluation in 1999-2000 from 140 excluded subjects with normal LFTs (including BMI, blood pressure, HOMA-IR, total cholesterol and triglycerides) [24].

The study was approved by the Ethics Committee at Lund University.

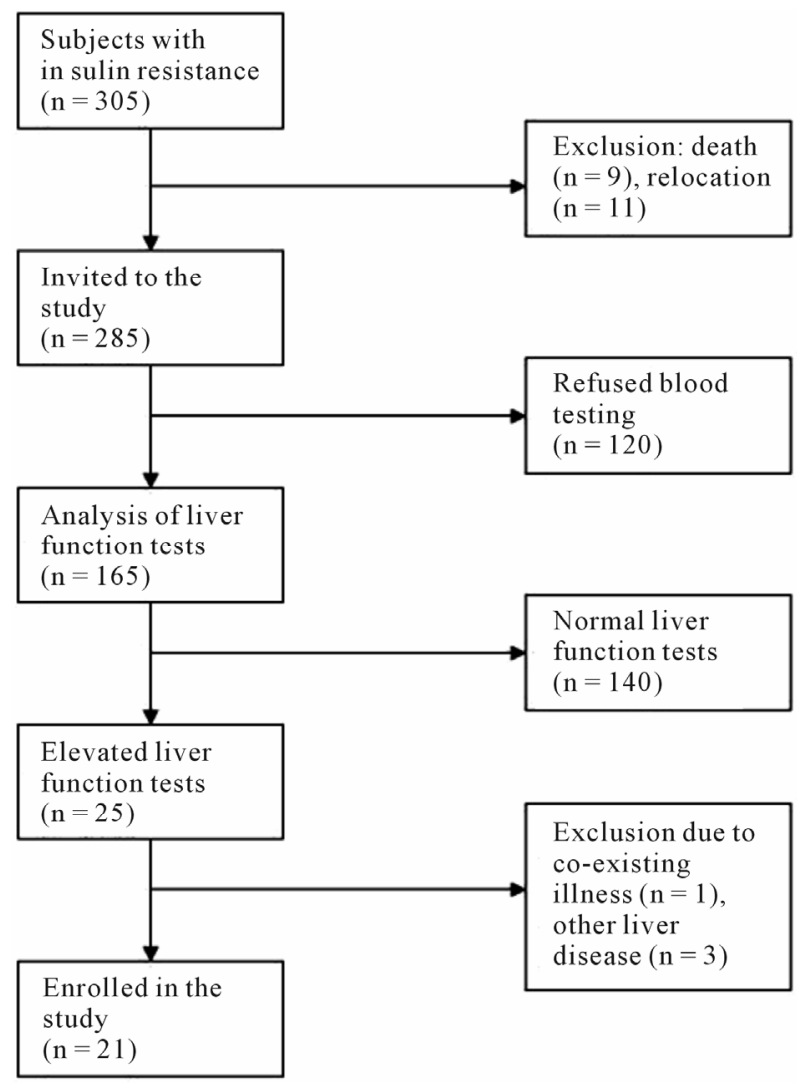

Figure 1. Method of inclusion of insulin resistant subjects with elevated liver enzymes and reasons for exclusion. 


\subsection{Baseline Examination}

Patients with elevated LFTs were invited to further assessment. Baseline examination was conducted by two physicians. Height $(\mathrm{cm})$ and weight $(\mathrm{kg})$ were measured, and BMI calculated $\left(\mathrm{kg} / \mathrm{m}^{2}\right)$. BMI $>25$ was classified as overweight and BMI $>30$ as obesity. Hip and waist circumference $(\mathrm{cm})$ were measured and waist-hip-ratio calculated. Blood pressure ( $\mathrm{mm} \mathrm{Hg}$ ) was measured twice in the supine position. A physical examination was performed. A complete medical history was obtained, including questions about alcohol intake. The average alcohol consumption was calculated in grams of ethanol per week and intake was assessed biochemically with CDT (carbohydrate-deficient transferrin). An ethanol consumption of $>140$ grams/week was regarded as over-consumption. Smoking was classified as never, former or current smokers. Biochemical analysis included a second control of LFTs (ALT, AST, gamma-GT, bilirubin, ALP and PKINR) together with total cholesterol, high density lipoprotein (HDL)-cholesterol and fasting triglycerides. Fasting plasma glucose and fasting serum insulin (enzyme immunometric assay) were analysed. Insulin resistance, indirectly measured as HOMA-IR, was re-calculated. To rule out other causes of chronic liver disease hepatitis $\mathrm{B}$ and $\mathrm{C}$ serology, serum iron, TIBC (total iron binding capacity), ferritin, serum gamma globulin (immunoglobulin $\mathrm{G}, \mathrm{A}$ and $\mathrm{M}$ ), ceruloplasmin, haptoglobin, alpha-1-antitrypsin and antimitochondrial (AMA), antinuclear (ANA) and smooth muscle (SMA) antibodies were analysed.

All blood tests were analysed in our central laboratory using standard reagents.

US or CT of the liver was performed and assessed regarding steatosis, focal changes, portal hypertension or other findings, indicating fibrosis or cirrhosis.

\subsection{Defining the Metabolic Syndrome}

The WHO clinical criteria for the metabolic syndrome was used, where insulin resistance (including established type 2 diabetes, impaired fasting glucose or glucose tolerance) co-exists with at least 2 of the following: Antihypertensive medication or high blood pressure (systolic blood pressure $\geq 140 \mathrm{~mm} \mathrm{Hg}$ and/or diastolic blood pressure $\geq 90 \mathrm{~mm} \mathrm{Hg})$, elevated plasma triglycerides $(\geq 1.7$ $\mathrm{mmol} / \mathrm{L})$ and/or low HDL cholesterol $(<0.9 \mathrm{mmol} / \mathrm{L}$ for men, $<1.0 \mathrm{mmol} / \mathrm{L}$ for women), BMI $>30$ and/or high waist-hip ratio ( $>0.90$ for men, $>0.85$ for women), and high urinary albumin excretion (not applicable in our study) $[6,7]$.

\subsection{Statistical Analysis}

SPSS Statistics version 19.0 was used for the statistical analyses. Categorical data are presented as number (per- centage). Continuous data are presented as mean \pm standard deviation (SD) and medians (range). Spearman's rank correlation coefficient was used for nonparametric measure of association. The non-parametric Mann-Whitney U-test was used to calculate significant differences between groups (NAFLD versus non-NAFLD). A p-value $<0.05$ was considered statistically significant.

\section{RESULTS}

\subsection{Patients}

305 subjects with insulin resistance were identified from the MDC cohort. After initial exclusion (Figure 1) 285 were invited by mail to leave blood samples for LFTs, which $165(57.9 \%)$ did. Of these, 25 (15\%) had elevated LFTs and were invited to further examination. One individual was later excluded due to suspected dementia, leaving 24 subjects enrolled for baseline examination. Two patients were excluded due to over-consumption of alcohol and one due to chronic hepatitis $\mathrm{C}$. The remaining 21 patients $(12.7 \%)$ were included in the statistical analysis (Table 1). Follow-up time was up to 17 years from the inclusion in the MDC cohort.

\subsection{Liver Complications}

All of the 21 patients underwent radiological examination of the liver (US or CT). Of these, $5(23.8 \%)$ had radio-

Table 1. Baseline characteristics of the enrolled study participants $(\mathrm{n}=21)$.

\begin{tabular}{|c|c|c|}
\hline & $\begin{array}{c}\text { Mean } \pm \mathrm{SD} \\
\mathrm{n}(\%)\end{array}$ & Median (range) \\
\hline Age (years) & $72.8 \pm 5.22$ & $74.0(61-78)$ \\
\hline Sex (female) & $16(76.2 \%)$ & - \\
\hline BMI & $28.6 \pm 4.66$ & $27.6(22.8-39.6)$ \\
\hline Waist-hip ratio & $0.91 \pm 0.079$ & $0.89(0.81-1.13)$ \\
\hline $\operatorname{AST}(\mathrm{U} / 1)^{\mathrm{a}}$ & $21.67 \pm 10.00$ & $17.06(11.76-42.35)$ \\
\hline $\operatorname{ALT}(\mathrm{U} / 1)^{\mathrm{a}}$ & $34.12 \pm 23.53$ & $24.12(11.76-100.00)$ \\
\hline Gamma-GT (U/1) ${ }^{\mathrm{a}}$ & $70.19 \pm 63.59$ & $44.39(15.00-281.34)$ \\
\hline Bilirubin $(\mu \mathrm{mol} / 1)$ & $15.6 \pm 4.79$ & $15.0(10.0-30.0)$ \\
\hline Glucose (mmol/l) & $5.95 \pm 1.04$ & $5.80(5.00-9.60)$ \\
\hline Insulin (mIU/l) & $10.3 \pm 4.55$ & $9.00(3.00-20.0)$ \\
\hline HOMA-IR & $2.78 \pm 1.48$ & $2.31(0.77-5.97)$ \\
\hline Total cholesterol (mmol/l) & $5.48 \pm 1.34$ & $5.20(3.80-8.90)$ \\
\hline HDL cholesterol (mmol/l) & $1.33 \pm 0.25$ & $1.28(1.00-1.96)$ \\
\hline Triglycerides (mmol/1) & $1.77 \pm 0.93$ & $1.50(0.70-3.80)$ \\
\hline Metabolic syndrome $^{\mathrm{b}}$ & $8(38.1 \%)$ & - \\
\hline Hypertension & $11(52.4 \%)$ & - \\
\hline Diabetes mellitus & $4(19.0 \%)$ & - \\
\hline Impaired fasting glucose $\mathrm{e}^{\mathrm{c}}$ & $3(14.2 \%)$ & - \\
\hline Overweight $(\mathrm{BMI}>25)$ & $16(76.2 \%)$ & - \\
\hline
\end{tabular}

${ }^{\mathrm{a} C}$ Conversion: ALT and ALT $(\mathrm{U} / \mathrm{l}) \times 0.017=\mu \mathrm{kat} / \mathrm{l}$. Gamma-GT $(\mathrm{U} / \mathrm{l}) \times$ $0.01667=\mu \mathrm{kat} / \mathrm{l}$. ${ }^{\mathrm{b}}$ According to the WHO criteria [7]. ${ }^{\mathrm{c}}$ Fasting plasma glucose $6.1-7 \mathrm{mmol} / 1$ 
logical signs of steatosis and were diagnosed with NAFLD.

None of the 21 patients had clinical signs or symptoms of chronic liver disease.

Liver steatosis significantly correlated with ALT ( $\mathrm{p}=$ 0.04), HOMA-IR $(\mathrm{p}=0.00)$ and the metabolic syndrome $(\mathrm{p}=0.03)$, when using non-parametric correlation test (Table 2).

When comparing the groups with and without NAFLD there was a significant difference in ALT $(p=0.04)$ and HOMA-IR $(\mathrm{p}=0.00)$, with higher values in the NAFLD group (Table 3 ).

\subsection{Metabolic Characteristics}

Since the re-investigation in 1999-2000 four patients (19\%) had been diagnosed with diabetes mellitus and three patients $(14.2 \%)$ had impaired fasting glucose $(6.1$ $7 \mathrm{mmol} / \mathrm{l}$ ) at enrolment (Table 1). In summary, 80\% of the patients with NAFLD had either developed diabetes mellitus or had impaired fasting glucose, compared to $18.8 \%$ in the group without NAFLD, a significant difference between the groups (Table 3).

Eight patients $(38.1 \%)$ were diagnosed with the metabolic syndrome and $50 \%$ of these were diagnosed with NAFLD. $80 \%$ of the patients with NAFLD met the criteria for the metabolic syndrome, compared to $25 \%$, also a significant difference between the groups (Table 3).

There was no significant difference in the occurrence of overweight and obesity between the patients with NAFLD and the patients without NAFLD.

\subsection{Excluded Patients}

None of the 284 patients who were excluded had an inpatient diagnosis of NAFLD. $2.1 \%$ had an inpatient diagnosis of diabetes mellitus type 2 and $1.8 \%$ had an inpatient liver diagnosis other than NAFLD.

When comparing anthropometric and laboratory data from the re-investigation in 1999-2000 between the included 21 subjects with elevated LFTs and the excluded 140 subjects with normal LFTs we found no significant differences in BMI, systolic or diastolic blood pressure, HOMA-IR, total cholesterol and triglycerides between the groups using the non-parametric Mann-Whitney Utest (data not shown).

\section{DISCUSSION}

Insulin resistance and NAFLD are closely linked. We have demonstrated that long-term insulin resistance alone carries a limited risk of at least moderate liver steatosis. The risk increases when associated with impaired fasting glucose or diabetes mellitus.

Only a minority of subjects (15\%) with insulin resistance had elevated liver function tests at long-term follow up. The frequency of NAFLD diagnosed radiologically in insulin resistant subjects with elevated liver enzymes, when the concomitant liver disease had been excluded, was $23.8 \%$ in our study. All of the affected subjects had either diabetes mellitus, impaired fasting glucose or fulfilled the criteria for the metabolic syndrome.

The strength of this study was the long follow-up time, in some cases up to 17 years. We also had a high re-

Table 2. Bivariate correlation between radiological steatosis and HOMA-IR, and clinical and laboratory features. Presented as Spearman's rho correlation coefficient and significance (two-tailed) at 0.05 level $\left({ }^{*}\right)$ and 0.01 level $\left({ }^{* *}\right)$.

\begin{tabular}{|c|c|c|c|c|}
\hline & \multirow{2}{*}{$\frac{\underline{\text { Steatosis }}}{\text { Spearman's rho }}$} & \multicolumn{3}{|c|}{$\underline{\text { HOMA-IR }}$} \\
\hline & & P-value & Spearman's rho & P-value \\
\hline BMI & 0.17 & 0.47 & 0.21 & 0.37 \\
\hline Metabolic syndrome $^{\mathrm{a}}$ & $0.48^{*}$ & 0.03 & 0.23 & 0.32 \\
\hline Waist $(\mathrm{cm})$ & $0.47^{*}$ & 0.03 & 0.28 & 0.22 \\
\hline Waist-hip ratio & 0.34 & 0.14 & $0.48^{*}$ & 0.03 \\
\hline AST (U/1) & 0.28 & 0.22 & $0.43^{*}$ & 0.05 \\
\hline $\operatorname{ALT}(\mathrm{U} / \mathrm{l})$ & $0.46^{*}$ & 0.04 & 0.37 & 0.11 \\
\hline Gamma-GT (U/1) & 0.33 & 0.14 & $0.47^{*}$ & 0.03 \\
\hline Bilirubin $(\mu \mathrm{mol} / \mathrm{l})$ & -0.04 & 0.87 & -0.35 & 0.12 \\
\hline Glucose (mmol/l) & 0.41 & 0.07 & $0.49^{*}$ & 0.03 \\
\hline Insulin (mIU/l) & $0.55^{* *}$ & 0.01 & $0.92^{* *}$ & 0.00 \\
\hline HOMA-IR & $0.65^{* *}$ & 0.00 & - & - \\
\hline Total cholesterol (mmol/l) & 0.32 & 0.15 & 0.19 & 0.40 \\
\hline HDL cholesterol (mmol/l) & $-0.43^{*}$ & 0.05 & 0.10 & 0.67 \\
\hline Triglycerides (mmol/l) & 0.22 & 0.33 & 0.24 & 0.24 \\
\hline
\end{tabular}

${ }^{a}$ Metabolic syndrome is defined according to the WHO criteria [7]. 
Table 3. Mann-Whitney U test comparing the groups steatosis $(n=5)$ vs. non-steatosis $(n=16)$.

\begin{tabular}{|c|c|c|c|c|}
\hline & Z-value & P-value & Mean rank steatosis & Mean rank non-steatosis \\
\hline Age & -0.91 & 0.36 & 8.80 & 11.69 \\
\hline Metabolic syndrome $^{\mathrm{a}}$ & -2.16 & 0.03 & 15.40 & 9.63 \\
\hline Diabetes mellitus & -1.33 & 0.18 & 13.20 & 10.31 \\
\hline $\mathrm{IFG}^{\mathrm{b}}$ & -1.83 & 0.07 & 13.70 & 10.16 \\
\hline Diabetes and $\mathrm{IFG}^{\mathrm{b}}$ & -2.48 & 0.01 & 15.90 & 9.47 \\
\hline BMI & -0.74 & 0.46 & 12.80 & 10.44 \\
\hline Waist (cm) & -2.11 & 0.04 & 16.10 & 9.41 \\
\hline Waist-hip ratio & -1.50 & 0.13 & 14.60 & 9.88 \\
\hline $\operatorname{ALT}(\mathrm{U} / \mathrm{l})$ & -2.07 & 0.04 & 16.00 & 9.44 \\
\hline $\operatorname{AST}(\mathrm{U} / \mathrm{l})$ & -1.25 & 0.21 & 14.00 & 10.06 \\
\hline Gamma-GT (U/l) & -1.49 & 0.14 & 14.60 & 9.88 \\
\hline Bilirubin $(\mu \mathrm{mol} / \mathrm{l})$ & -0.17 & 0.87 & 10.60 & 11.13 \\
\hline Insulin (mIU/l) & -2.46 & 0.01 & 16.90 & 9.16 \\
\hline Glucose (mmol/l) & -1.82 & 0.07 & 15.40 & 9.63 \\
\hline HOMA-IR & -2.89 & 0.00 & 18.00 & 8.81 \\
\hline Total cholesterol (mmol/l) & -1.45 & 0.15 & 14.50 & 9.91 \\
\hline HDL cholesterol (mmol/l) & -1.94 & 0.05 & 6.30 & 12.47 \\
\hline Triglycerides (mmol/l) & -0.99 & 0.32 & 13.40 & 10.25 \\
\hline
\end{tabular}

${ }^{\mathrm{a}}$ Metabolic syndrome is defined according to the WHO criteria [7]; ${ }^{\mathrm{b}}$ Impaired fasting glucose (fasting plasma glucose $6.1-7 \mathrm{mmol} / \mathrm{l}$ ).

sponse rate at nearly $60 \%$ at invitation. However, the study is limited by a small sample size.

One would expect a higher frequency of NAFLD in a high risk population of insulin resistant subjects than in the general population. We defined NAFLD as a positive radiological finding meaning that at least $20 \%-30 \%$ of the liver is composed of fat $[8,9]$. Histologically steatosis is commonly evaluated semi-quantitatively assessing percentage involvement by steatotic hepatocytes: score 1 (mild, 5\% - 33\%), 2 (moderate, $33 \%-66 \%$ ) and 3 (severe, $>66 \%$ ) [15]. With US and CT we have been able to diagnose patients with moderate and severe steatosis. There is of course a possibility that the frequency of NAFLD could be higher if patients had been biopsied. In a previous study by Mathiesen et al., it was also conducted in Sweden the prevalence of steatosis was $40 \%$ among subjects who underwent liver biopsy due to asymptomatic slightly-moderately elevated LFTs of all causes [25]. More newer sensitive non-invasive techniques like magnetic resonance spectroscopy have come to clinical usage after this study is designed, which could strengthen the results it had been used. Still it is obvious that insulin resistance alone does not lead to liver steatosis in the majority of patients.
The role of insulin resistance in NAFLD is complex. Both hepatic and systemic insulin resistance are strongly associated with NAFLD. Visceral fat may be of higher importance than whole body fat, possibly since the former is released directly into the portal vein. In an insulin resistant state, inability of insulin to suppress lipolysis leads to increased flux of free fatty acids to the liver from adipose tissue. Increased de novo lipogenesis and increased intake of dietary fat contribute to the development of NAFLD. In a fatty liver the ability of insulin to inhibit hepatic glucose production is impaired leading to hyperglycemia and further insulin resistance [21,26-28]. In our study waist circumference, which is a surrogate marker of visceral adiposity, the occurrence of diabetes mellitus/ impaired fasting glucose and the metabolic syndrome was significantly higher in subjects with NAFLD.

One limitation to the interpretation of the role of insulin resistance is the measure of HOMA-IR, an approximation of insulin resistance often used in large epidemiological studies. HOMA-IR reflects the balance between insulin secretion and hepatic glucose output in a basal fasting state. The model has been compared to well-validated methods including euglycemic clamp, and there is a good correlation between these tests. Insulin secretion, 
however, is pulsatile, and a single measurement is not as reliable as a mean of three [29]. There is no world-wide standardized cut-off level for HOMA-IR in diagnosing insulin resistance and the insulin assay differs between countries. According to the WHO criteria for the metabolic syndrome, insulin resistance is defined as the lowest $25 \%$ glucose uptake in the background population in the hyperinsulinemic euglycemic clamp [6,7]. In our study all the included subjects were in the upper quartile of HOMA-IR in a previous study from the original MDC cohort and were classified as insulin resistant [23]. They had significantly higher BMI, waist circumference and blood lipids than the lowest quartile. Patients with previously known diabetes mellitus were excluded from our study and many had died from cardiovascular disease, leaving a selection of more healthy subjects to be enrolled in our study. However, this rather strengthens the focus on insulin resistance per se.

Insulin resistance may change over time. Recently, a large Korean prospective study among healthy subjects also explored the risk of developing NAFLD in non-diabetic subjects. After 5 years 13\% had developed NAFLD, diagnosed with ultrasound. These subjects were older, more obese, had higher insulin levels and liver enzymes at baseline and follow-up than subjects who did not develop NAFLD. Subjects with high insulin levels at baseline and low at follow-up had a similar risk of developing NAFLD as subjects with sustained low levels of fasting insulin [30]. In our study five subjects were at enrolment now below the previous gender-specific $75^{\text {th }}$ percentile of HOMA-IR in the MDC cohort (i.e. 1.80 for women, 2.12 for men, adjusting for fasting plasma glucose to the previous method of fasting blood glucose), and none of these subjects developed NAFLD, which corresponds to the results in the Korean study.

Another limitation is that subjects with insulin resistance and normal LFTs might also have NAFLD. ALT is a commonly used surrogate marker in screening for liver disease. In the majority of cases with NAFLD an asymptomatic elevation of LFTs is found and previous studies have shown an association between ALT and NAFLD [10]. In our study we confirmed previous results and found a significant correlation between NAFLD and ALT. The entire spectrum of NAFLD can occur despite normal LFTs, however, the grade of steatosis has been shown to correlate to higher ALT $[11,12]$. Ekstedt et al. showed in a previous Swedish study that patients with biopsy-proven NAFLD and elevated LFTs had a risk of developing end-stage liver disease at long-term follow-up [31]. Patients with more progressive liver disease had a higher prevalence of insulin resistance, quantitatively more steatosis, more weight gain and higher LFTs than patients with a more benign course. It is therefore unlikely that we have missed a large proportion of individuals with significant and severe NAFLD among the ones with normal liver enzymes. Using the Swedish National Board of Health and Welfare's National Patient Register for inpatient diagnoses we did not find any excluded patients (including patients with normal LFTs) who had been diagnosed with NAFLD and the prevalence of diabetes mellitus was very low.

We found no significant differences regarding BMI, blood pressure, HOMA-IR or lipids at the re-evaluation in 1999-2000 among the subjects who were excluded due to normal LFTs $(n=140)$ and the included patients $(n=$ 21). Longstanding insulin resistance measured as HOMAIR seems therefore not to be enough to develop elevated LFTs and NAFLD. Progression of insulin resistance to impaired fasting glucose and eventually established diabetes mellitus seems to be of importance in the pathogenesis of NAFLD, as indicated in our study, possibly because of a combination of systemic and hepatic insulin resistance.

In conclusion, the risk of having at least moderate liver steatosis after more than a decade of insulin resistance is not insignificant, but mainly a risk if associated with established diabetes mellitus, impaired fasting glucose and/or the metabolic syndrome.

\section{REFERENCES}

[1] Angulo, P. (2002) Nonalcoholic fatty liver disease. New England Journal of Medicine, 346, 1221-1231. doi:10.1056/NEJMra011775

[2] Sass, D.A., Chang, P. and Chopra, K.B. (2005) Nonalcoholic fatty liver disease: A clinical review. Digestive Diseases and Sciences, 50, 171-180. doi:10.1007/s10620-005-1267-z

[3] Blachier, M., Leleu, H., Peck-Radosavljevic, M., Valla, D.C. and Roudot-Thoraval, F. (2013) The burden of liver disease in Europe: A review of available epidemiological data. Journal of Hepatology, 58, 593-608. doi:10.1016/j.jhep.2012.12.005

[4] Torres, D.M., Williams, C.D. and Harrison, S.A. (2012) Features, diagnosis, and treatment of nonalcoholic fatty liver disease. Clinical Gastroenterology and Hepatology, 10, 837-858. doi:10.1016/j.cgh.2012.03.011

[5] Marchesini, G., Bugianesi, E., Forlani, G., Cerrelli, F., Lenzi, M., Manini, R., et al. (2003) Nonalcoholic fatty liver, steatohepatitis, and the metabolic syndrome. Нераtology, 37, 917-923. doi:10.1053/jhep.2003.50161

[6] Balkau, B., Charles, M.A., Drivsholm, T., Borch-Johnsen, K., Wareham, N., Yudkin, J.S., et al. (2002) Frequency of the WHO metabolic syndrome in European cohorts, and an alternative definition of an insulin resistance syndrome. Diabetes \& Metabolism, 28, 364-376.

[7] Eckel, R.H., Grundy, S.M. and Zimmet, P.Z. (2005) The metabolic syndrome. Lancet, 365, 1415-1428. doi:10.1016/S0140-6736(05)66378-7

[8] Hernaez, R., Lazo, M., Bonekamp, S., Kamel, I., Brancati, 
F.L., Guallar, E., et al. (2011) Diagnostic accuracy and reliability of ultrasonography for the detection of fatty liver: A meta-analysis. Hepatology, 54, 1082-1090. doi:10.1002/hep. 24452

[9] Schwenzer, N.F., Springer, F., Schraml, C., Stefan, N., Machann, J. and Schick, F. (2009) Non-invasive assessment and quantification of liver steatosis by ultrasound, computed tomography and magnetic resonance. Journal of Hepatology, 51, 433-445. doi:10.1016/i.jhep.2009.05.023

[10] Schindhelm, R.K., Diamant, M., Dekker, J.M., Tushuizen, M.E., Teerlink, T. and Heine, R.J. (2006) Alanine aminotransferase as a marker of non-alcoholic fatty liver disease in relation to type 2 diabetes mellitus and cardiovascular disease. Diabetes/Metabolism: Research and Reviews, 22, 437-443. doi:10.1002/dmrr.666

[11] Fracanzani, A.L., Valenti, L., Bugianesi, E., Andreoletti, M., Colli, A., Vanni, E., et al. (2008) Risk of severe liver disease in nonalcoholic fatty liver disease with normal aminotransferase levels: A role for insulin resistance and diabetes. Hepatology, 48, 792-798. doi:10.1002/hep.22429

[12] Mofrad, P., Contos, M.J., Haque, M., Sargeant, C., Fisher, R.A., Luketic, V.A., et al. (2003) Clinical and histologic spectrum of nonalcoholic fatty liver disease associated with normal ALT values. Hepatology, 37, 1286-1292. doi:10.1053/jhep.2003.50229

[13] Bugianesi, E., Leone, N., Vanni, E., Marchesini, G., Brunello, F., Carucci, P., et al. (2002) Expanding the natural history of nonalcoholic steatohepatitis: From cryptogenic cirrhosis to hepatocellular carcinoma. Gastroenterology, 123, 134-140. doi:10.1053/gast.2002.34168

[14] Caldwell, S.H. and Crespo, D.M. (2004) The spectrum expanded: Cryptogenic cirrhosis and the natural history of non-alcoholic fatty liver disease. Journal of Hepatology, 40, 578-584. doi:10.1016/j.jhep.2004.02.013

[15] Brunt, E.M. and Tiniakos, D.G. (2010) Histopathology of nonalcoholic fatty liver disease. World Journal of Gastroenteroly, 16, 5286-5296. doi:10.3748/wjg.v16.i42.5286

[16] Dam-Larsen, S., Franzmann, M., Andersen, I.B., Christoffersen, P., Jensen, L.B., Sorensen, T.I., et al. (2004) Long term prognosis of fatty liver: Risk of chronic liver disease and death. Gut, 53, 750-755. doi:10.1136/gut.2003.019984

[17] Farrell, G.C. and Larter, C.Z. (2006) Nonalcoholic fatty liver disease: From steatosis to cirrhosis. Hepatology, 43, S99-S112. doi:10.1002/hep.20973

[18] Haque, M. and Sanyal, A.J. (2002) The metabolic abnormalities associated with non-alcoholic fatty liver disease. Best Practice \& Research Clinical Gastroenterology, 16, 709-731. doi:10.1053/bega.2002.0325

[19] Day, C.P. (2002) Pathogenesis of steatohepatitis. Best Practice \& Research Clinical Gastroenterology, 16, 663-678. doi:10.1053/bega.2002.0333

[20] Fracanzani, A.L., Valenti, L., Bugianesi, E., Vanni, E., Grieco, A., Miele, L., et al. (2011) Risk of nonalcoholic stea- tohepatitis and fibrosis in patients with nonalcoholic fatty liver disease and low visceral adiposity. Journal of Hepatology, 54, 1244-1249. doi:10.1016/j.jhep.2010.09.037

[21] Eguchi, Y., Mizuta, T., Sumida, Y., Ishibashi, E., Kitajima, Y., Isoda, H., et al. (2011) The pathological role of visceral fat accumulation in steatosis, inflammation, and progression of nonalcoholic fatty liver disease. Journal of Gastroenterology, 46, 70-78. doi:10.1007/s00535-010-0340-3

[22] Bonora, E., Kiechl, S., Willeit, J., Oberhollenzer, F., Egger, G., Targher, G., et al. (1998) Prevalence of insulin resistance in metabolic disorders: The Bruneck Study. Diabetes, 47, 1643-1649. doi:10.2337/diabetes.47.10.1643

[23] Hedblad, B., Nilsson, P., Engstrom, G., Berglund, G. and Janzon, L. (2002) Insulin resistance in non-diabetic subjects is associated with increased incidence of myocardial infarction and death. Diabetic Medicine, 19, 470-475. doi:10.1046/j.1464-5491.2002.00719.x

[24] Nilsson, P.M., Engstrom, G., Hedblad, B., Frystyk, J., Persson, M.M., Berglund, G., et al. (2006) Plasma adiponectin levels in relation to carotid intima media thickness and markers of insulin resistance. Arteriosclerosis, Thrombosis and Vascular Biology, 26, 2758-2762. doi:10.1161/01.ATV.0000249638.01416.4b

[25] Mathiesen, U.L., Franzen, L.E., Fryden, A., Foberg, U. and Bodemar, G. (1999) The clinical significance of slightly to moderately increased liver transaminase values in asymptomatic patients. Scandinavian Journal of Gastroenterology, 34, 85-91. doi: $10.1080 / 00365529950172880$

[26] Bugianesi, E., McCullough, A.J. and Marchesini, G. (2005) Insulin resistance: A metabolic pathway to chronic liver disease. Hepatology, 42, 987-1000. doi:10.1002/hep.20920

[27] Leclercq, I.A., Da Silva Morais, A., Schroyen, B., Van Hul, N. and Geerts, A. (2007) Insulin resistance in heaptocytes and sinusoidal liver cells: mechanisms and consequences. Journal of Hepatology, 47, 142-156. doi:10.1016/j.jhep.2007.04.002

[28] Tilg, H. and Moschen, A.R. (2008) Insulin resistance, inflammation, and non-alcoholic fatty liver disease. Trends in Endocrinology \& Metabolism, 19, 371-379. doi:10.1016/j.tem.2008.08.005

[29] Wallace, T.M., Levy, J.C. and Matthews, D.R. (2004) Use and abuse of HOMA modeling. Diabetes Care, 27, 1487-1495. doi:10.2337/diacare.27.6.1487

[30] Rhee, E.J., Lee, W.Y., Cho, Y.K., Kim, B.I. and Sung, K.C. (2011) Hyperinsulinemia and the development of nonalcoholic Fatty liver disease in nondiabetic adults. American Journal of Medicine, 124, 69-76. doi:10.1016/j.amjmed.2010.08.012

[31] Ekstedt, M., Franzen, L.E., Mathiesen, U.L., Thorelius, L., Holmqvist, M., Bodemar, G., et al. (2006) Long-term follow-up of patients with NAFLD and elevated liver enzymes. Hepatology, 44, 865-873. doi:10.1002/hep. 21327 